\title{
Genomic Characterization of a Novel Gut Symbiont From the Hadal Snailfish
}

\author{
Chun-Ang Lian ${ }^{1,2}$, Guo-Yong Yan ${ }^{1,2}$, Jiao-Mei Huang ${ }^{1,2}$, Antoine Danchin ${ }^{3}$, Yong Wang ${ }^{1 *}$ \\ and Li-Sheng He ${ }^{1 *}$ \\ ${ }^{1}$ Institute of Deep-Sea Science and Engineering, Chinese Academy of Sciences, Sanya, China, ${ }^{2}$ College of Earth Sciences, \\ University of Chinese Academy of Sciences, Beijing, China, ${ }^{3}$ Department of Infection, Immunity and Inflammation, \\ Institut Cochin, INSERM U1016 - CNRS UMR 8104 - Université Paris Descartes, Paris, France
}

\section{OPEN ACCESS}

Edited by:

Jean-Christophe Avarre, Institut de Recherche pour le Développement (IRD), France

Reviewed by:

Paul Yancey,

Whitman College, United States

Nan Peng,

Huazhong Agricultural University,

China

*Correspondence:

Yong Wang

wangy@idsse.ac.cn

Li-Sheng $\mathrm{He}$

he-lisheng@idsse.ac.cn

Specialty section:

This article was submitted to

Aquatic Microbiology,

a section of the journal

Frontiers in Microbiology

Received: 14 August 2019 Accepted: 10 December 2019 Published: 10 January 2020

Citation:

Lian C-A, Yan G-Y, Huang J-M, Danchin A, Wang Y and He L-S (2020) Genomic Characterization of a Novel Gut Symbiont From the Hadal Snailfish. Front. Microbiol. 10:2978. doi: 10.3389/fmicb.2019.02978
Hadal trenches are characterized by not only high hydrostatic pressure but also scarcity of nutrients and high diversity of viruses. Snailfishes, as the dominant vertebrates, play an important role in hadal ecology. Although studies have suggested possible reasons for the tolerance of hadal snailfish to high hydrostatic pressure, little is known about the strategies employed by hadal snailfish to cope with low-nutrient and virus-rich conditions. In this study, the gut microbiota of hadal snailfish was investigated. A novel bacterium named "Candidatus Mycoplasma liparidae" was dominant in the guts of three snailfish individuals from both the Mariana and Yap trenches. A draft genome of "Ca. Mycoplasma liparidae" was successfully assembled with $97.8 \%$ completeness by hybrid sequencing. A set of genes encoding riboflavin biosynthesis proteins and a clustered regularly interspaced short palindromic repeats (CRISPR) system was present in the genome of "Ca. Mycoplasma liparidae," which was unusual for Mycoplasma. The functional repertoire of the "Ca. Mycoplasma liparidae" genome is likely set to help the host in riboflavin supplementation and to provide protection against viruses via a super CRISPR system. Remarkably, genes encoding common virulence factors usually exist in Tenericutes pathogens but were lacking in the genome of "Ca. Mycoplasma liparidae." All of these characteristics supported an essential role of "Ca. Mycoplasma liparidae" in snailfish living in the hadal zone. Our findings provide further insights into symbiotic associations in the hadal biosphere.

Keywords: hadal symbiosis, Tenericutes, CRISPR, snailfish, metagenome

\section{INTRODUCTION}

The hadal zone, at water depths below 6,000 m, comprises mainly trenches that plunge from the abyssal plains to depths of up to $11,000 \mathrm{~m}$ and is the least explored region on Earth (Somero, 1992; Jamieson et al., 2010). In contrast to the upper abyssal oceans, the trench environment represents a distinct and often extreme deep habitat with some striking features. The primary characteristic of the hadal zone is high hydrostatic pressure, which increases with depth, reaching up to 1.1 tons per square centimeter in the deepest ocean trenches (Jamieson et al., 2010; Nunoura et al., 2015). Moreover, the light intensity in the hadal zone is too low to sustain photosynthetic primary production, and the availability of organic matter that is mainly supplemented by surface-derived carrion falls controls benthic biomass (Jorgensen and Boetius, 2007). However, most of the 
sinking material is consumed and intercepted by plankton and heterotrophic bacteria in shallower and bathyal waters that selectively remove highly labile compounds before the material reaches the trenches. Thus, trench communities are typically considered nutrition-limited systems (Jamieson et al., 2010). In addition, deep-sea ecosystems are rich with viruses, which are a predominant cause of the mortality of microorganisms, which in turn contribute to biogeochemical cycles (Hara et al., 1996; Yoshida et al., 2013; Anantharaman et al., 2014). In deep-sea environments, prokaryotic mortality due to viruses is estimated to be dozens of times more than that due to other causes (Lara et al., 2017). Although the hadal environment is extreme, diverse organisms inhabit this zone. How these creatures survive in such extreme conditions is still unclear. Sampling difficulties are the main reasons, and studies on these topics have progressed very slowly. Fishes are the only vertebrates inhabiting the hadal zone, and snailfishes are the dominant ichthyofauna at depths of approximately 6,000-8,145 $\mathrm{m}$ (Stein, 2005; Linley et al., 2016). A previous study showed that the osmolyte trimethylamine $N$-oxide (TMAO) helped snailfish stabilize proteins against pressure (Yancey et al., 2014). With the interpretation of the hadal snailfish genome, the mechanism underlying the adaptation of hadal snailfish to pressure has been understood from a new perspective (Wang et al., 2019). However, with regard to the characteristics of the hadal environment, in addition to pressure, nutrient acquisition, micronutrient biosynthesis and immune protection are also critical for organismal survival due to the low nutrient levels under normal conditions and the high abundance of unknown viruses and other potentially harmful microorganisms in the surrounding water compared to the surface (Hara et al., 1996; Yoshida et al., 2013).

The gut is a very important organ for the survival and health of organisms in many respects. Almost all of these biological activities are accomplished with the assistance of intestinal microbes (Ley et al., 2008; Nicholson et al., 2012; Douglas, 2015; Paniagua Voirol et al., 2018). This phenomenon is particularly evident for organisms in deep sea. In the stomach microbiota of Bathynomus sp., a deep-sea isopod living at a depth of $898 \mathrm{~m}$ in the South China Sea, symbiotic mycoplasmas occupy $42.8-100 \%$ of the microbial communities and might provide sugars and amino acids to the hosts (Wang et al., 2016). A sea cucumber from a depth of $>6,000 \mathrm{~m}$ in the Mariana Trench harbored obligate symbiotic spiroplasmas, with $63.5 \%$ occupation in the hindgut bacterial community. Spiroplasma symbionts might be able to protect the host against viral infections through clustered regularly interspaced short palindromic repeats (CRISPR) systems (He et al., 2017). For the hadal dominant species Hirondellea gigas, Tenericutes were also found in the gut microbial communities in the Mariana Trench (Zhang et al., 2018). Distinct from these deep-sea scavengers, the hadal snailfish primarily feeds on amphipods (Gerringer et al., 2017b), serving as a major player in deep-sea ecosystems and on the top of the food chain. The microbial inhabitants in the snailfish gut and their contributions to host survival remain unknown. In this study, we will characterize a novel mycoplasma, the dominant microbe in the hadal snailfish gut, and describe its contributions to hadal snailfish survival.

\section{MATERIALS AND METHODS}

\section{Sample Collection and Preparation}

Two hadal snailfishes were collected from the Mariana Trench (141 ${ }^{\circ} 56.8663^{\prime} \mathrm{E}, 10^{\circ} 59.2497^{\prime} \mathrm{N}$; sample IDs: MT1 and MT2) at a depth of 7,034 $\mathrm{m}$ on 19th and 28th of July 2016 by the research ship "Tan Suo Yi Hao," and one was obtained from the Yap Trench $\left(138^{\circ} 32.3808^{\prime} \mathrm{E}, 9^{\circ} 43.6578^{\prime} \mathrm{N}\right.$; sample ID: YT1) at a depth of 7,884 $\mathrm{m}$ on 23rd of February 2017 also by “Tan Suo Yi Hao." The locations for sampling are mapped in Supplementary Figure S1. The hadal snailfishes were trapped with baits using a sampler installed on a lander. The bait was wrapped with a high density sack to avoid direct ingestion. All of the hadal snailfishes were classified as Pseudoliparis swirei based on COI and morphology (Gerringer et al., 2017a). For comparison, two shallow-water snailfishes Liparis tanakae were collected from the southern central Yellow Sea by trawling at a depth of $20 \mathrm{~m}$ in November 2016. Specimens were dissected immediately once captured on board. To compare the microbial community structure in different gut segments, the guts were isolated and divided into three segments including front (Fgut), middle (Mgut), and hind (Hgut) segments, accordingly. Samples for molecular studies were stored at $-80^{\circ} \mathrm{C}$ until use. Total DNA was extracted using PowerSoil DNA Isolation Kit (QIAGEN, United States). The quality and quantity of the extracted DNA were checked by gel electrophoresis and Qubit 2.0 Fluorometer (Life Technologies, United States), respectively.

\section{Cloning and Classification of 16S rRNA Gene Sequences}

The full-length $16 \mathrm{~S}$ rRNA genes of the microbes from snailfish guts were amplified with a pair of universal primers (27F: 5'-AGAGTTTGATC[C/A]TGGCTCAG-3'; 1492R: 5'TACGG[C/T]TACCTTGTTACGAC-3') (Wang et al., 2016). PCR amplification was performed in a $50 \mu \mathrm{l}$ reaction containing $5 \mathrm{ng}$ of bacterial DNA as template, $10 \mu \mathrm{l}$ of $5 \times$ PCR buffer, $4 \mu \mathrm{l}$ of $10 \mathrm{mM}$ dNTPs, $0.5 \mu \mathrm{l}$ of Prime STAR HS DNA Polymerase (TaKaRa, Japan), $32.5 \mu \mathrm{l}$ of $\mathrm{ddH}_{2} \mathrm{O}$, and $2 \mu \mathrm{l}$ of $10 \mu \mathrm{M}$ each primer. The amplification program consisted of an initial denaturation step at $98^{\circ} \mathrm{C}$ for $10 \mathrm{~s}$, followed by 25 cycles of $98^{\circ} \mathrm{C}$ for $10 \mathrm{~s}, 55^{\circ} \mathrm{C}$ for $15 \mathrm{~s}$, and $72^{\circ} \mathrm{C}$ for $90 \mathrm{~s}$, with a final extension of $10 \mathrm{~min}$ at $72^{\circ} \mathrm{C}$. A no-template control was used to check for possible contaminations. The PCR products were separated by gel electrophoresis, and then the bands with expected size $(\sim 1,500 \mathrm{bp})$ were purified with a QIAquick purification kit (Qiagen, Germany). After ligations into a vector pMD18-T (TaKaRa, Japan), the products were transformed into JM109 competent cells (TaKaRa, Japan). Thirty positive clones were randomly collected for each sample and sequenced using a Sanger sequencer (ABI 3730, United States). The chimera sequences were removed according to the previous study (Haas et al., 2011). Taxonomic assignment was based on the Silva online tools with default settings ${ }^{1}$. The classified sequences were further checked by searching against the NCBI database.

\footnotetext{
${ }^{1}$ https://www.arb-silva.de
} 


\section{Hybrid Sequencing, Metagenomic Assembly, Binning, and Annotation}

A total of $200 \mathrm{ng}$ of genomic DNA from the MT1 Hgut was employed for a paired-end library with insert size of $550 \mathrm{bp}$ using TruSeq DNA Sample Prep Kit (Illumina, United States). The above library was sequenced twice on an Illumina MiSeq platform using V3 Miseq sequencing kits $(2 \times 300 \mathrm{bp})$ (Illumina, United States) to ensure sufficient data; 43.1 million $300 \mathrm{bp}$ paired-end metagenomic reads were generated. Raw reads were processed with the NGS QC Toolkit version 2.3.3 (Patel and Jain, 2012 ) to remove short ( $<70 \mathrm{bp}$ ) and low quality (quality score $<20$ ) reads, followed by trimming adaptor sequences. Finally, 38.3 million clean reads were generated. Nanopore libraries were generated using 1D^2 sequencing kit (Oxford Nanopore, United Kingdom) with $1.5 \mu \mathrm{g}$ of genomic DNA, and then sequenced using the Nanopore MinION system. 3.6 million long Nanopore reads ( $>4 \mathrm{~kb}$ ) were generated. Metagenomic assembly of the Illumina paired-end reads and Nanopore reads was performed using SPAdes 3.11 (Bankevich et al., 2012), with a maximum k-mer length of 127. The Mycoplasma draft genome was binned through separation based on different coverage levels and the $\mathrm{G}+\mathrm{C}$ contents of the metagenomic contigs. The coverage levels of the assembled contigs were calculated using the idxstats module of SAMtools version 0.1.191 (Li et al., 2009). The binning process was visualized using RStudio ${ }^{2}$ according to a previous study (Albertsen et al., 2013). The coding sequences (CDSs) were predicted using Prodigal version 2.60 (Land et al., 2010), and configured with -g 4 and -p meta. The tRNA genes in the genome were identified using tRNAscan 1.3.1 (Lowe and Eddy, 1997). The quality and completeness of the draft genome were determined using CheckM (version 1.0.5) with the lineage workflow (Parks et al., 2015).

The annotation of deduced proteins was performed using BLASTp against four databases including NCBI Nr, KEGG (Ogata et al., 2000), Pfam (Finn et al., 2014), and COG (Sverdlov et al., 2003) with a maximum e-value cutoff of 1e-04. OrthoFinder 2.2.3 (Emms and Kelly, 2015) was used to further examine the shared and genome-specific genes among different bacteria with the default settings. The CRISPR region was identified using the CRISPRFinder online tool (Grissa et al., 2007).

\section{Reconstruction of Phylogenetic Trees}

The 16S rRNA sequences used for the phylogenetic trees were obtained from NCBI and EzTaxon websites. Eight sequences from genus Acholeplasma, 22 from Entomoplasmatales, six from Hepatoplasma, nine from Ureaplasma, and 25 from Mycoplasma were selected for the phylogenetic tree with five Bacillus sequences used as the outgroup. The accession numbers are shown following the species names. Alignment was performed using MUSCLE3.5 with manual adjustments (Edgar, 2004), and a maximum-likelihood (ML) phylogenetic tree with 1,000 bootstraps was subsequently constructed using raxmlGUI (Silvestro and Michalak, 2012) with a GTRGAMMA model. The $16 \mathrm{~S}$ rRNA sequences of mycoplasmas from different

${ }^{2}$ https://www.rstudio.com
P. swirei individuals were also used to analyze the phylogenetic relationships with the same method. In addition, 54 Tenericutes genomes (including the current Mycoplasma genome) belonging to different taxonomic groups were pooled. Conserved singlecopy genes (CSCGs) were identified by searching the CDSs against the Pfam database using Hmmsearch 3.0 with the default settings (Finn et al., 2014). A phylogenomic analysis was conducted using a total of 20 CSCGs (Supplementary Table S1) from a set of 31 genes previously defined for bacterial phylogenomic inference (Wu and Eisen, 2008). These 20 CSCGs were shared by all of the 54 genomes. Each of the 20 CSCGs was individually aligned with MUSCLE 3.5. The resulting aligned files were concatenated using a custom Perl script, and the resulting supermatrix was processed to estimate ML phylogeny with raxmlGUI with 1,000 bootstraps using PROTGAMMALG model, according to the previous study (He et al., 2017).

\section{Multilocus Gene Sequencing}

The bacterial housekeeping genes (atpA, $\operatorname{rec} A$, and $g y r B$ ) were selected (Polzin et al., 2019) for PCR amplifications with a set of custom primers (Supplementary Table S2). DNA extracted from the guts was used as template. PCR reactions consisted of $50 \mu \mathrm{l}$ of $1 \mu \mathrm{l}$ DNA, $10 \mu \mathrm{l}$ of $5 \times$ PCR buffer, $4 \mu \mathrm{l}$ of $10 \mathrm{mM}$ dNTPs, $0.5 \mu \mathrm{l}$ of Prime STAR HS DNA Polymerase (TaKaRa, Japan), $32.5 \mu \mathrm{l}$ of $\mathrm{ddH}_{2} \mathrm{O}$, and $2 \mu \mathrm{l}$ of $10 \mu \mathrm{M}$ each primer. The following program was applied: $98^{\circ} \mathrm{C}$ for $10 \mathrm{~s} ; 30$ cycles of $98^{\circ} \mathrm{C}$ for $10 \mathrm{~s}$, annealing for $15 \mathrm{~s}, 72^{\circ} \mathrm{C}$ for $2 \mathrm{~min}$, and a final extension of $10 \mathrm{~min}$ at $72^{\circ} \mathrm{C}$. Annealing temperature was specific to each primer set (Supplementary Table S2). The PCR products were observed on $1 \%$ agarose gel electrophoresis. The amplicons with a correct size were sequenced using a Sanger sequencer (ABI 3730, United States).

\section{RESULTS}

\section{Gut Microbiota of the Snailfishes}

Gut microbial communities in snailfishes were revealed by fulllength $16 \mathrm{~S}$ rRNA sequences $(\sim 1,500 \mathrm{bp})$. A total of 270 clones from three $P$. swirei individuals were examined and classified into five phyla including Proteobacteria, Firmicutes, Tenericutes, Bacteroidetes, Planctomycetes, and unclassified one. In P. swirei Fguts, Firmicutes ranked the first with relative abundance of $35.0 \pm 9.4 \%$. For the second and third top phyla, Proteobacteria and Tenericutes occupied $21.7 \pm 12.1 \%$ and $16.0 \pm 2.9 \%$ of the microbial communities, respectively. However, P. swirei Mguts and Hguts were dominated by Tenericutes with relative abundances of $31.2-64.5 \%$ (Figure 1). The second and third top phyla were Firmicutes (18.8-29.2\%) and Proteobacteria (6.7$21.6 \%)$. Moreover, Mycoplasma was the only genus from the Tenericutes phylum. The $16 \mathrm{~S}$ rRNA sequences of mycoplasmas from different gut segments or different $P$. swirei individuals were nearly identical (>99.9\% similarity). The relationship between different individuals was confirmed by phylogenetic analysis (Supplementary Figure S2). However, the gut microbiota of the two shallow-water snailfishes, L. tanakae, was dominated by the 
genera Clostridium and Psychrobacter, while Mycoplasma was absent (Supplementary Figure S3).

\section{Genome Sequence of Mycoplasma}

Based on the analysis of gut microbiota, Mycoplasma was the dominant microbe in P. swirei guts, especially in Mguts and Hguts. To obtain more genomic information about the dominant Mycoplasma, metagenomic sequencing was performed. After optimized pipelines for assembly, the sequencing coverage for the assembled contigs, GC content, and taxonomic affiliation of the CSCGs derived from the contigs were analyzed in the RStudio platform with the libraries of vegan, plyr, RColorBrewer, and alphahull to classify these contigs into different genomes. Then, a draft genome of 795,917 bp for the dominant Mycoplasma was achieved at the coverage level of approximately 20 -fold, with an extremely low G + C content of 23.5\% (Table 1 and Supplementary Figure S4). The Mycoplasma of snailfish was $97.8 \%$ complete and included three contigs, 750 predicted protein-coding genes, 95 CSCGs, 30 tRNA genes, and a complete set of tRNA genes for 20 amino acids. To further investigate whether the dominant Mycoplasma species from P. swirei guts were polyclonal or monoclonal, the housekeeping genes atpA, $r e c A$, and $g y r B$ were amplified and sequenced from the three P. swirei Hguts. The three housekeeping gene sequences revealed no variation (100\% identity) across all the Hguts. These results indicated that a monoclonal Mycoplasma strain might exist in the P. swirei guts.
Compared with the other two genomes of dominant Tenericutes from the deep-sea cucumber and the isopod Bathynomus sp. (Table 1), Mycoplasma of snailfish was larger in size, while coding density was slightly sparse. In terms of metabolic pathways, Mycoplasma of snailfish contained more genes encoding riboflavin metabolism and $\mathrm{ABC}$ transporters, but had fewer phosphotransferase transport systems than mycoplasma BG1.

\section{Phylogenetic Analysis}

A total of 70 sequences from five groups in Tenericutes were used to construct a phylogenetic tree. The current Mycoplasma was grouped within the pneumoniae group and approximated to a symbiotic Mycoplasma from the goby gut $(97 \%$ identity for $16 \mathrm{~S}$ rRNA sequences), which was supported by a bootstrap value of 100 (Figure 2). Compared with Mycoplasma muris, the snailfish Mycoplasma was more distantly related to members of Ureaplasma. The high genetic relatedness of snailfish Mycoplasma with other Mycoplasma species was further substantiated by a phylogenomic analysis using 53 additional genomes from different taxonomic groups in Tenericutes (Supplementary Figure S5). Phylogenetic analysis revealed that snailfish Mycoplasma represents a novel lineage between Mycoplasma group and Ureaplasma group. Thus, the species named "Candidatus Mycoplasma liparidae" is proposed for this bacterium (hereafter, genome of "Candidatus Mycoplasma liparidae"; CML).

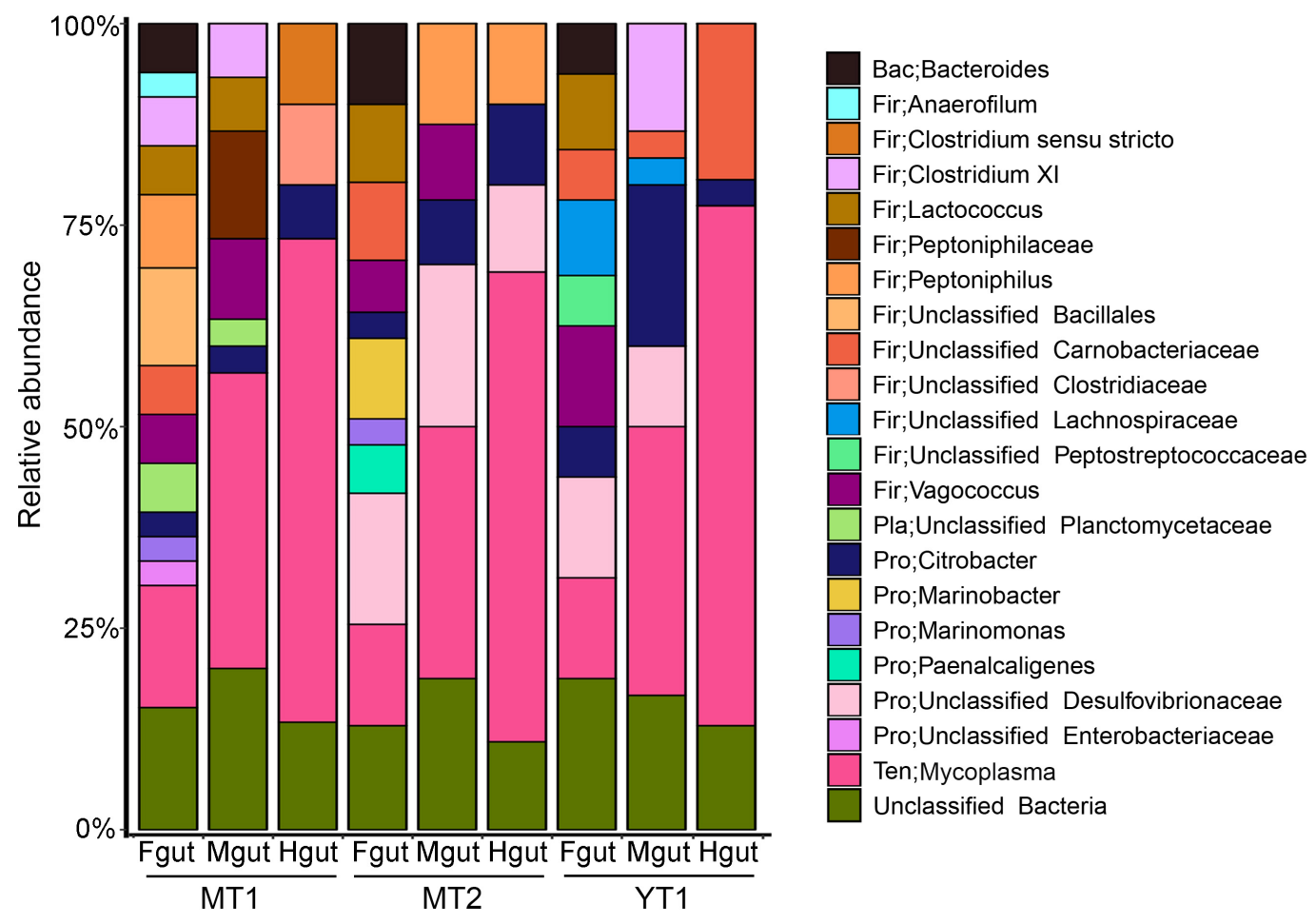

FIGURE 1 | Bacterial communities in the hadal snailfish gut. A total of 270 clones for $16 S$ rRNA sequences were randomly collected and sequenced. The Silva database was used as a reference for classification with default settings. Fgut: front segment of the gut; Mgut: middle segment of the gut; Hgut: hind segment of the gut; MT: snailfishes from the Mariana Trench; YT: snailfish from the Yap Trench. 
TABLE 1 | Genome features and KEGG pathways of deep-sea symbiotic Tenericutes.

\begin{tabular}{lccc}
\hline Genome features and KEGG pathways & CML & CSH & MB \\
\hline Genome size (bp) & 795,917 & 424,539 & 785,028 \\
Contig number & 3 & 2 & 5 \\
GC content (\%) & 23.5 & 29.6 & 26.5 \\
CDS number & 750 & 347 & 678 \\
tRNA genes & 30 & 32 & 33 \\
rRNA genes & 3 & 2 & 3 \\
Coding density (\%) & 86.8 & 90.2 & 91.8 \\
Conserved single-copy genes & 95 & 98 & 101 \\
CheckM completeness (\%) & 97.8 & $/$ & $/$ \\
CheckM contamination (\%) & 0.0 & $/$ & $/$ \\
ko00220 Arginine biosynthesis & 3 & 3 & 3 \\
ko00740 Riboflavin metabolism & 7 & 1 & 2 \\
ko02010 ABC transporters & 12 & 6 & 10 \\
ko02060 Phosphotransferase system & 1 & 0 & 12 \\
References & This study & He et al., & Wang et al., \\
& & 2017 & 2016 \\
\hline
\end{tabular}

CML: the genome of "Ca. Mycoplasma liparidae" (PRJNA497967); CSH: "Ca. Spiroplasma holothuricola" (CP022928/9), a hadal holothurian symbiont; MB: mycoplasma BG1 (LSMX00000000), isolated from a deep-sea isopod.

To further understand the relationship between " $\mathrm{Ca}$. Mycoplasma liparidae" and other Tenericutes, we selected 29 Tenericutes genomes to calculate the average nucleotide identity (ANI). The ANI value ranged from 60.9 to $65.5 \%$ with a standard deviation of $0.79 \%$ (Supplementary Table S3), showing that the closest known relative of " $\mathrm{Ca}$. Mycoplasma liparidae" was Mycoplasma iowae.

\section{Metabolic Features}

In the CML, a single phosphotransferase system (PTS) was identified (Figure 3). But, CML did not possess genes involved in pyruvate degradation and tricarboxylic acid cycle (Figure 3). "Ca. Mycoplasma liparidae" may produce ATP through the fermentation of arginine (arginolysis). All the genes encoding the enzymes that took part in arginolysis via the arginine deiminase system (ADI pathway) were identified in the CML (Figure 3). The arginine deiminase gene $(\operatorname{arc} A)$ in the CML shared its highest identity (70\%) to Mycoplasma moatsii. Carbamoyl-P, a product of citrulline degradation, is likely catabolized by carbamate kinase (Ckase) to generate ATP, ammonia, and $\mathrm{CO}_{2}$. However, only one gene $(g p m I)$ was involved in glycine, serine, and threonine metabolism; six genes ( $m e t K$, four $d n m T$ and $m t n N$ ) were involved in methionine metabolism, but the key genes (metA, met $B$, met $C$, and met $E$ ) of methionine biosynthesis were lost; one gene $(d a p F)$ was responsible for lysine biosynthesis; four genes (four $\operatorname{aguA}$ ) were involved in proline metabolism; and one gene (katA) was involved in tryptophan metabolism. No gene was related to the metabolism of the remaining amino acids. Amino acid biosynthetic genes were almost entirely eliminated, suggesting that " $\mathrm{Ca}$. Mycoplasma liparidae" may obtain amino acids from other sources, such as the host.

Notably, CML was found to harbor all of the key genes involved in the biosynthesis of riboflavin (vitamin $\mathrm{B}_{2}$ ), including ribA, $r i b D, r i b Z(y c s E), r i b B A, r i b H$, and $r i b E$ (Figure 3).

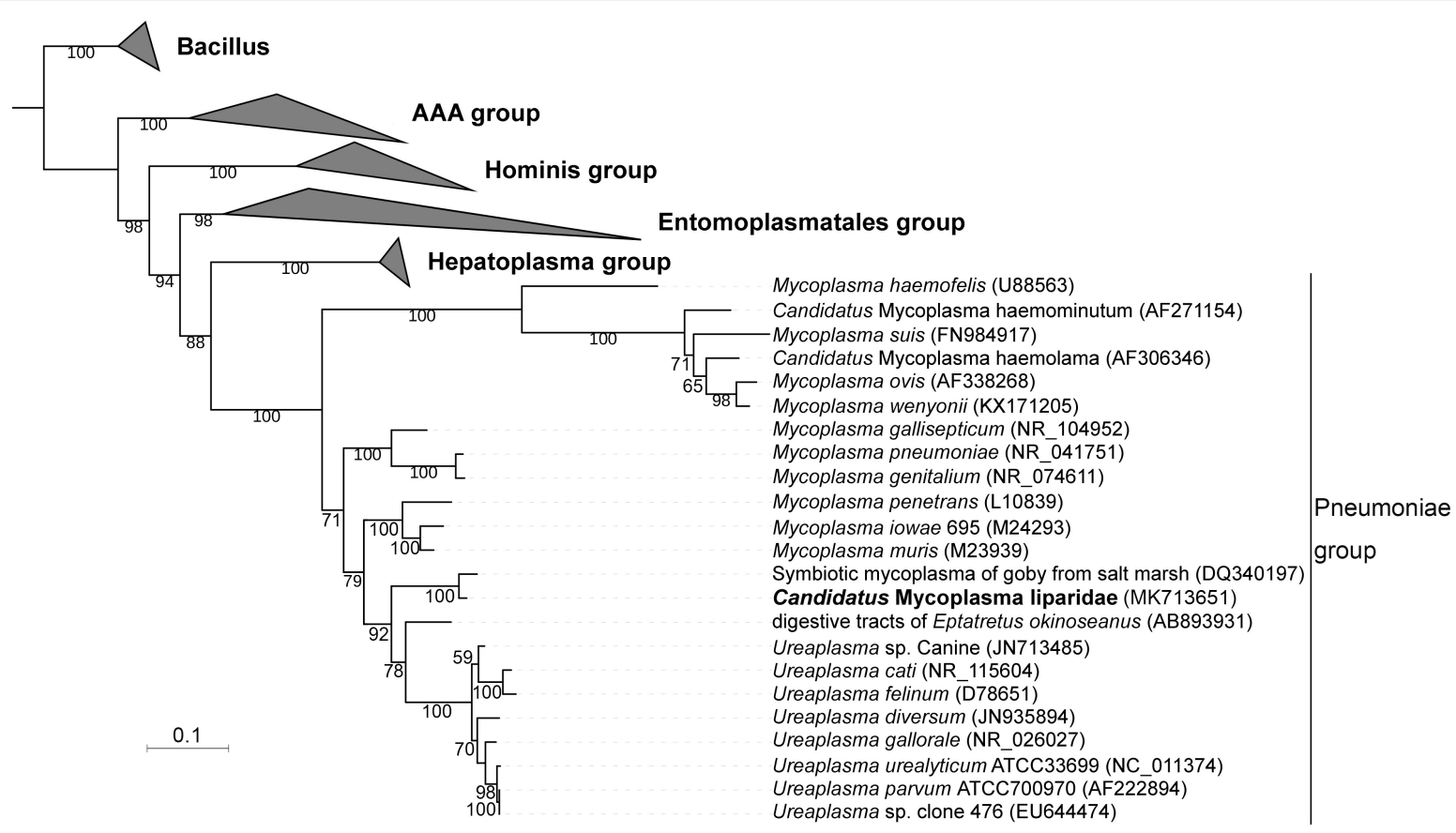

FIGURE 2 | Phylogenetic position of "Ca. Mycoplasma liparidae." The 16S rRNA sequence of "Ca. Mycoplasma liparidae" was aligned with those of 69 Tenericutes to reconstruct a maximum-likelihood tree with 1,000 replications. The bootstrap values are indicated on the branches of the tree. If a sequence is not associated with a species name, the host and source are indicated. The species investigated in this study is highlighted. 


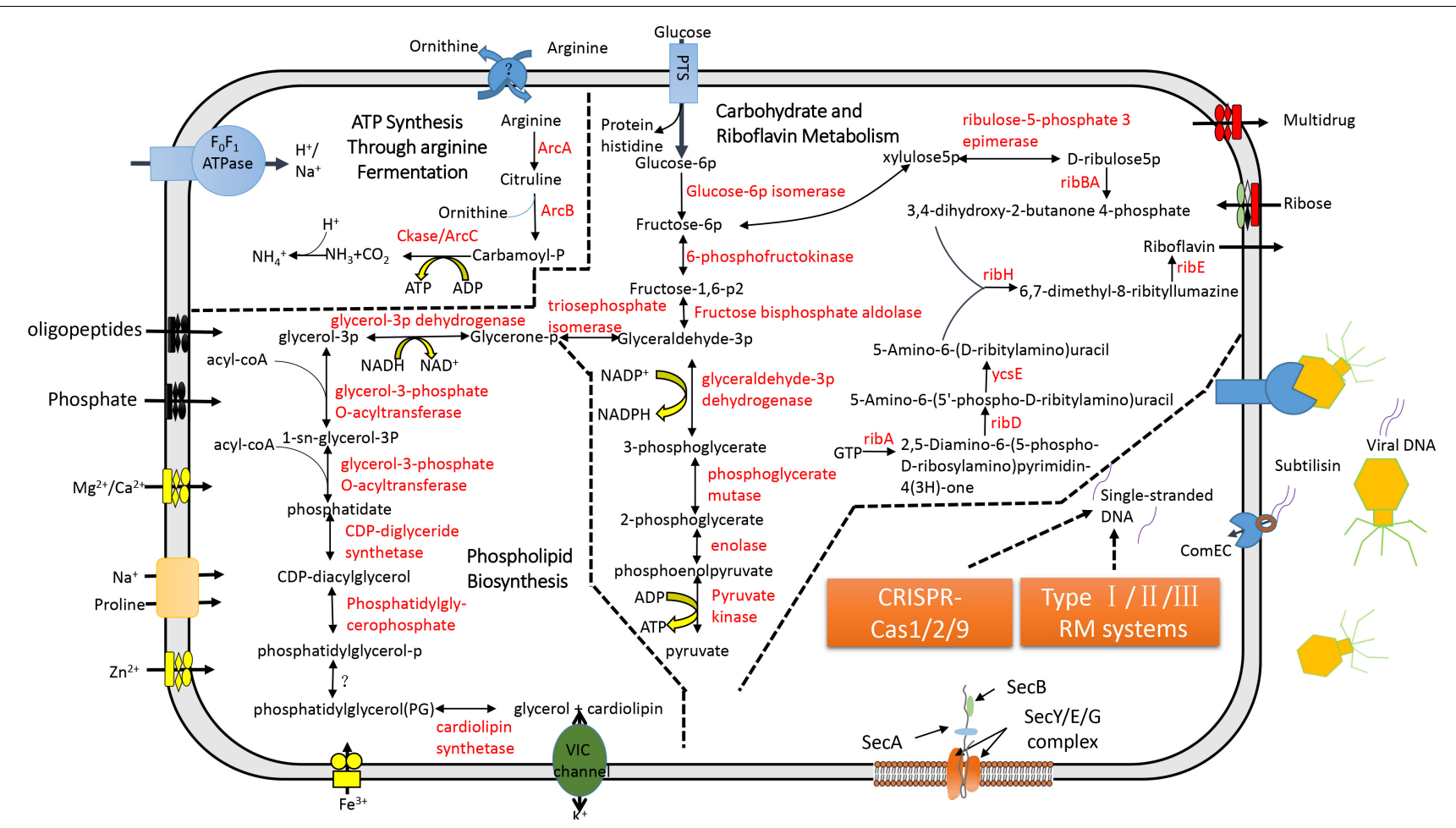

FIGURE 3 | Metabolic pathways and the CRISPR-Cas system in "Ca. Mycoplasma liparidae." The primary metabolic pathways in "Ca. Mycoplasma liparidae" were predicted in an integrated manner. Metabolic products are shown in black, and enzymes or proteins are shown in red. Question marks denote transporters or enzymes that were not identified.

A comparison of the gene order at the corresponding genome region in the six genomes showed that $r i b D$, $r i b E$, ribBA, and ribH usually clustered together and were conservatively present (Supplementary Figure S6A). Riboflavin synthase (ribE), a critical enzyme, catalyzes the final step in the riboflavin biosynthetic pathway to yield riboflavin. The riboflavin synthase in " $\mathrm{Ca}$. Mycoplasma liparidae" has not been reported in Mollicutes. The most similar homolog was from a Clostridium sp. CAG (49\% identity at amino acid level). The phylogenetic tree revealed that CML-derived riboflavin synthase branched together with that in Clostridium sp. CAG (Supplementary Figure S6B). To examine the presence of a binding site of the substrate for riboflavin synthase in "Ca. Mycoplasma liparidae," the alignment of amino acid sequences was performed with three wellcharacterized homologs to show the conserved sites and motifs (Supplementary Figure S7).

\section{Absence of Virulence Factors}

The CML lacks a $g l p D$ gene, which is the key gene for $\mathrm{H}_{2} \mathrm{O}_{2}$ production via a glycerol catabolic pathway, but encodes a catalase $(54 \%$ identity to the homolog of $M$. iowae) for $\mathrm{H}_{2} \mathrm{O}_{2}$ degradation. To gain further insight into the genetic disparity between "Ca. Mycoplasma liparidae" and pathogenic bacteria, we compared the gene content of " $\mathrm{Ca}$. Mycoplasma liparidae" with that of four pathogens, namely, Ureaplasma urealyticum, Ureaplasma diversum, Mycoplasma gallisepticum, and Mycoplasma pneumoniae, all belonging to the pneumoniae group. Their core genome comprised 233 genes (Figure 4). As expected, the functions of these common genes were concentrated in translation, ribosomal structure, and biogenesis. Nevertheless, the genomes of the four pathogens have some particular genes encoding multiple-banded antigen, IgA protease, urease, ADP-ribosylating, and other virulence factors. All of these virulence genes were absent from CML (Supplementary Table S4). Intriguingly, CML harbored 315 unique genes, of which only 69 genes had KEGG orthology. A total of 171 genes corresponded to proteins with an unknown function.

\section{Immune Protection}

Here, a CRISPR-Cas system consisting of three cas genes (cas $1 /$ cas $2 /$ cas 9 ) and 118 spacers were detected in CML. Homologous genes with the highest identity were found in the genomes of M. moatsii ( $48 \%$ for cas1; $44 \%$ for cas 2 ) and Mycoplasma alvi (28\% for cas9). However, an unusual organization of CRISPR-Cas genes was displayed in CML compared to that of $M$. gallisepticum. The cas 9 gene was located downstream of the CRISPR locus and was in a reverse orientation (Figure 5).

To identify the matching viruses, the sequences of CRISPR spacers were searched against the virus collection in the NCBI database (NCBI taxid 10,239), and the following viruses and phages were identified: $0.85 \%$ of the spacers (one 


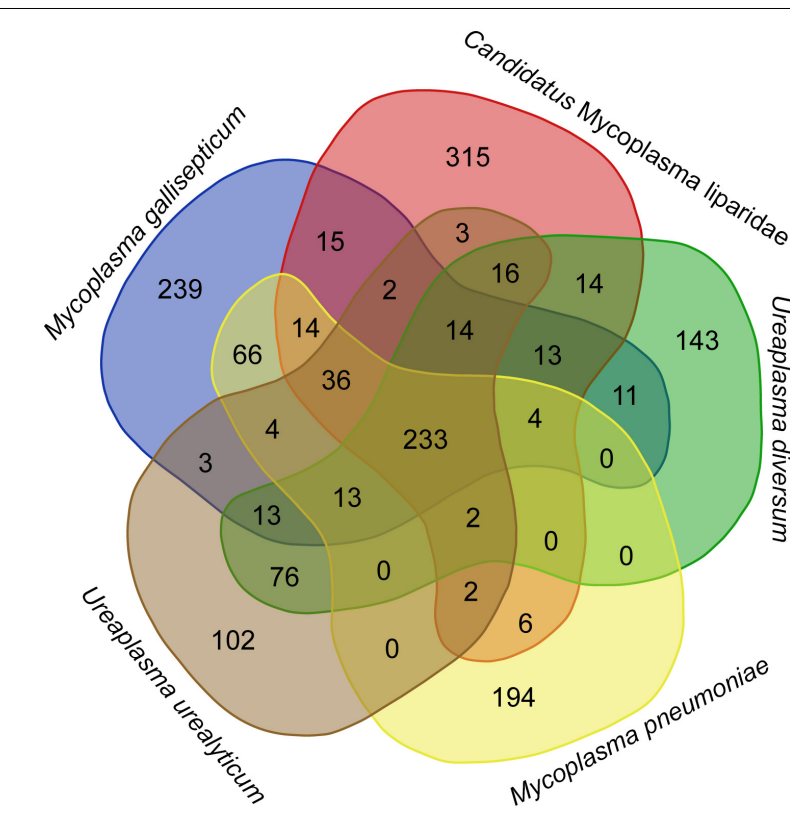

FIGURE 4 | Diagram showing shared and species-specific gene clusters. Gene content was compared among "Ca. Mycoplasma liparidae" (PRJNA497967), U. urealyticum (NC_011374), U. diversum (CP009770), M. gallisepticum (NC_004829), and M. pneumoniae (NC_000912).

Orthogroup analysis was used to show the common genes between these bacterial genomes.

spacer) matched Acanthamoeba castellanii mimivirus (100\% identity in nucleotide sequence), $0.85 \%$ of the spacers matched Chrysochromulina ericina virus (93\% identity), and $0.85 \%$ of the spacers matched Megavirus (100\% identity); $2.54 \%$ of the spacers matched Lactococcus phage (92\% identity), $4.23 \%$ of the spacers matched Bacillus phage (92\% identity), $2.54 \%$ of the spacers matched Vibrio phage (95\% identity), and $0.85 \%$ of the spacers matched Hydrogenobaculum phage (92\% identity) (Supplementary Table S5).

\section{DISCUSSION}

In this study, three $P$. swirei individuals from two trenches displayed the same taxa, "Ca. Mycoplasma liparidae," in the guts, which were indicated to be the same strain by analyses of the 16S rRNA gene and three housekeeping genes. The presence of several similar bacterial taxa in the gut-associated microbiota of the same or multiple fish species from different geographical locations indicated that these ribotypes might be important contributors to gut functions (Roeselers et al., 2011; Ghanbari et al., 2015), referred to as the "core gut microbiota" (Rawls et al., 2004; Kessel et al., 2011; Wong et al., 2013). Such a gut symbiosis system was restricted to hadal snailfish species, as we did not detect a similar gut microbiota in shallow-sea snailfish. In "Ca. Mycoplasma liparidae," glucose can be transported from outside and degraded into pyruvate via the Embden-MeyerhofParnas pathway (Robillard and Broos, 1999). However, due to absence of oxidative phosphorylation systems, arginolysis may be an alternative process for the generation of ATP (Vander et al., 1984) in "Ca. Mycoplasma liparidae."

The genome of the hadal snailfish $P$. swirei revealed no genes associated with riboflavin biosynthesis (Wang et al., 2019), but a complete set of genes involved in riboflavin biosynthesis was present in the genome of " $\mathrm{Ca}$. Mycoplasma liparidae," indicating that $P$. swirei may largely depend on "Ca. Mycoplasma liparidae" for the supply of riboflavin when it was insufficient in foods. Riboflavin biosynthesis occurs in many bacteria, such as Bacillus subtilis and Escherichia coli (Leblanc et al., 2013), but is quite unusual in Tenericutes. To the best of our knowledge, this is the first report to show that Mycoplasma can synthesize riboflavin. Riboflavin synthase is the key enzyme for riboflavin biosynthesis and has no homolog in other Tenericutes but shares 49\% identity with Clostridium, suggesting the occurrence of horizontal gene transfer. As expected, "Ca. Mycoplasma liparidae" and Clostridium sp. CAG were placed into one clade by phylogenetic analysis using riboflavin synthases. In addition, the alignment of amino acid sequences with three well-characterized homologs (Eberhardt et al., 1996) indicates the presence of binding sites

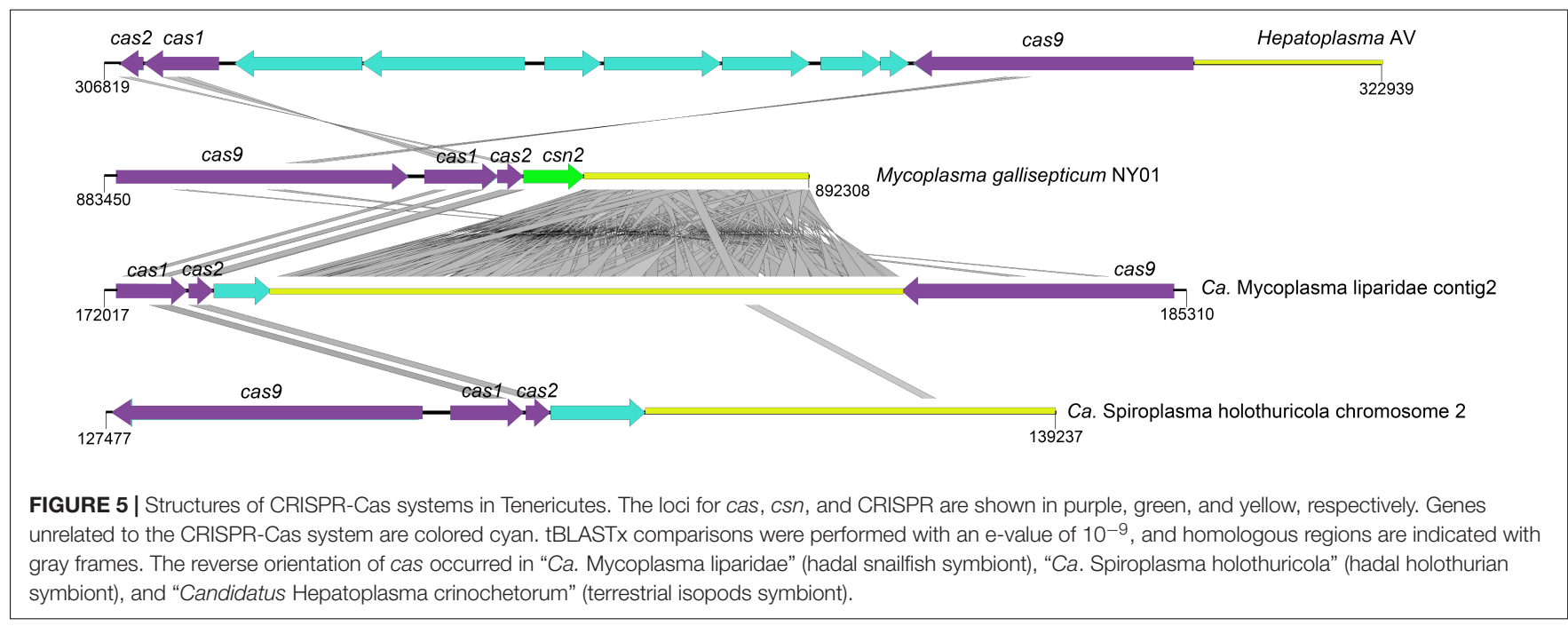


in riboflavin synthase of CML. Instead, virulence factors were absent in the CML, but common to other pathogens (Glass et al., 2000; Kannan and Baseman, 2006; Szczepanek et al., 2010; Indikova et al., 2013; Kokkayil and Dhawan, 2015) and genes involved in the generation of $\mathrm{H}_{2} \mathrm{O}_{2}$, which is a major mediator for Mycoplasma pathogens to cause cellular damage (Pritchard et al., 2014), were also absent in the CML. All these results indicated that " $\mathrm{Ca}$. Mycoplasma liparidae" co-evolved with host and other bacteria during symbiosis. Phylogenetic trees using both the 16S rRNA genes and genomes showed that " $\mathrm{Ca}$. Mycoplasma liparidae" was almost equidistant from Mycoplasma and Ureaplasma, which is not a novel phenomenon. It has been reported that symbiotic Mycoplasma from the digestive tracts of scorpions represent a novel Mycoplasma group according to 16S rRNA sequence analysis (Elmnasri et al., 2018); symbionts of termites have been classified as a new lineage within the Mycoplasma genus (Hongoh et al., 2003). Not limited to Tenericutes, intracellular symbionts residing in the bacteriomes of leafhoppers fell into a well-defined clade within Proteobacteria (Moran et al., 2003). Similarly, the Spiroplasma symbiont from the gut of the hadal sea cucumber was distantly related to known species (He et al., 2017), probably due to genetic drift during a long-term symbiosis.

Deep-sea waters and sediments are replete with viruses (Hara et al., 1996; Danovaro et al., 2008). The development of an efficient immune system to address this situation is the key to survival. CRISPR-Cas system confers adaptive immunity for prokaryotes against mobile elements and phage infections (Deveau et al., 2010; Barrangou and Marraffini, 2014). In this study, the CRISPR system encoded by CML may prevent viral infections to protect snailfishes in three aspects: (i) against phages to maintain normal gut microbiota; (ii) against viruses infecting the host; and (iii) against viruses infecting the gut parasite that potentially regulates homeostasis of the host. It is possible that the one end of "Ca. Mycoplasma liparidae" may be exposed to the outside of the gut epithelial cell, suggesting its ability to capture and degrade some phages in the snailfish gut, and the other end of "Ca. Mycoplasma liparidae" enters the inside of the gut cell, underlying it could probably destroy the viruses infecting the host. "Ca. Mycoplasma liparidae," which may be partially embedded in the gut wall, resembles the human pathogen Mycoplasma genitalium, which infects lung cells (Jensen et al., 1994). At present, several CRISPR-Cas subtypes have been identified, each with a specific set of cas genes (Makarova et al., 2015). The cas 9 gene in the CML is a type II CRISPR-Cas subtype, usually composed of cas9, cas1, cas2, and cas4 (for type IIB) or csn2 (for type IIA) genes or only three cas genes (for type IIC), all located in a single transcriptional unit directly upstream of the CRISPR locus (Zhang et al., 2013; Makarova et al., 2015). Interestingly, the cas9 gene was located downstream of the CRISPR locus and was in a reverse orientation in the "Ca. Mycoplasma liparidae" genome. The reverse orientation of the cas gene has also been observed in symbionts of hadal holothurian and terrestrial isopods. In addition, all of the above three symbionts lacked the csn 2 gene. This type of CRISPR-Cas system with cas gene rearrangements was thought to be largely inefficient to against further infections (Leclercq et al., 2014).
However, type IIC systems that stimulate immunity without the $\operatorname{csn} 2$ gene have been proposed (Zhang et al., 2013; Chylinski et al., 2014). A recent study reported a new subtype CRISPR-Cas system lacking the $\operatorname{csn} 2$ gene in an uncultivated archaeal strain (Burstein et al., 2017), in which the locus organization was similar to that of "Ca. Mycoplasma liparidae." Our findings indicated that the cas gene rearrangements may point toward the evolution of a new CRISPR-Cas subtype.

\section{DATA AVAILABILITY STATEMENT}

Sequencing data has been deposited in the NCBI GenBank. The CML has been deposited at GenBank under the BioProject accession number: PRJNA497967. The 16S rRNA gene sequence is accessible in GenBank under the accession number: MK713651.

\section{ETHICS STATEMENT}

Mariana and Yap hadal snailfish specimens were trapped using deep-sea landers carried by the research ship "Tan Suo Yi Hao." The captured hadal snailfishes were dead once collected on deck. Wild shallow-water snailfishes were collected from a depth of $20 \mathrm{~m}$ in the southern central Yellow Sea by trawling. All of the snailfishes are neither endangered nor protected animals. Animal sampling was carried out in accordance with the animal experimentation guidelines and regulations of the Institute of Deep-Sea Science and Engineering. The protocol was approved by the Federated States of Micronesia, with permit numbers FM16-CN20162RS-01 and FM17-CN20162RS-01, respectively.

\section{AUTHOR CONTRIBUTIONS}

C-AL, YW, and L-SH conceived and designed the experiments. G-YY, AD, and J-MH performed the experiments and analyzed the data. C-AL and L-SH wrote the manuscript with input from all other members. YW and L-SH directed and supervised all of the research.

\section{FUNDING}

This study was supported by the Key R\&D Program of China (Grant Nos. 2018YFC0309804, 2016YFC0302504, and 2018YFC0310005) and the Strategic Priority Research Program $\mathrm{B}$ of the Chinese Academy of Sciences (CAS) (Grant Nos. XDB06010103 and XDB06010201).

\section{SUPPLEMENTARY MATERIAL}

The Supplementary Material for this article can be found online at: https://www.frontiersin.org/articles/10.3389/fmicb. 2019.02978/full\#supplementary-material 


\section{REFERENCES}

Albertsen, M., Hugenholtz, P., Skarshewski, A., Nielsen, K. L., Tyson, G. W., and Nielsen, P. H. (2013). Genome sequences of rare, uncultured bacteria obtained by differential coverage binning of multiple metagenomes. Nat. Biotechnol. 31, 533-538. doi: 10.1038/nbt.2579

Anantharaman, K., Duhaime, M. B., Breier, J. A., Wendt, K. A., Toner, B. M., and Dick, G. J. (2014). Sulfur oxidation genes in diverse deep-sea viruses. Science 344, 757-760. doi: 10.1126/science.1252229

Bankevich, A., Nurk, S., Antipov, D., Gurevich, A. A., Dvorkin, M., Kulikov, A. S., et al. (2012). SPAdes: a new genome assembly algorithm and its applications to single-cell sequencing. J. Comput. Biol. 19, 455-477. doi: 10.1089/cmb.2012. 0021

Barrangou, R., and Marraffini, L. A. (2014). CRISPR-Cas systems: prokaryotes upgrade to adaptive immunity. Mol. Cell. 54, 234-244. doi: 10.1016/j.molcel. 2014.03.011

Burstein, D., Harrington, L. B., Strutt, S. C., Probst, A. J., Anantharaman, K., Thomas, B. C., et al. (2017). New CRISPR-Cas systems from uncultivated microbes. Nature 542, 237-241. doi: 10.1038/nature21059

Chylinski, K., Makarova, K. S., Charpentier, E., and Koonin, E. V. (2014). Classification and evolution of type II CRISPR-Cas systems. Nucleic Acids Res. 42, 6091-6105. doi: 10.1093/nar/gku241

Danovaro, R., Dell'Anno, A., Corinaldesi, C., Magagnini, M., Noble, R., Tamburini, C., et al. (2008). Major viral impact on the functioning of benthic deep-sea ecosystems. Nature 454, 1084-1087. doi: 10.1038/nature07268

Deveau, H., Garneau, J. E., and Moineau, S. (2010). CRISPR/Cas system and its role in phage-bacteria interactions. Annu. Rev. Microbiol. 64, 475-493. doi: 10.1146/annurev.micro.112408.134123

Douglas, A. E. (2015). Multiorganismal insects: diversity and function of resident microorganisms. Annu. Rev. Entomol. 60, 17-34. doi: 10.1146/annurev-ento010814-020822

Eberhardt, S., Richter, G., Gimbel, W., Werner, T., and Bacher, A. (1996). Cloning, sequencing, mapping and hyperexpression of the ribC gene coding for riboflavin synthase of Escherichia coli. Eur. J. Biochem. 242, 712-719. doi: 10.1111/j.1432-1033.1996.0712r.x

Edgar, R. C. (2004). MUSCLE: multiple sequence alignment with high accuracy and high throughput. Nucleic Acids Res. 32, 1792-1797. doi: 10.1093/nar/gkh340

Elmnasri, K., Hamdi, C., Ettoumi, B., Crotti, E., Guesmi, A., Najjari, A., et al. (2018). Highly divergent Mollicutes symbionts coexist in the scorpion Androctonus australis. J. Basic. Microb. 58, 827-835. doi: 10.1002/jobm.2018 00144

Emms, D. M., and Kelly, S. (2015). OrthoFinder: solving fundamental biases in whole genome comparisons dramatically improves orthogroup inference accuracy. Genome Biol. 16:157. doi: 10.1186/s13059-015-0721-2

Finn, R. D., Bateman, A., Clements, J., Coggill, P., Eberhardt, R. Y., Eddy, S. R., et al. (2014). Pfam: the protein families database. Nucleic Acids Res. 42, D222-D230. doi: 10.1093/nar/gkt1223

Gerringer, M. E., Linley, T. D., Jamieson, A. J., Goetze, E., and Drazen, J. C. (2017a). Pseudoliparis swirei sp. nov.: a newly-discovered hadal snailfish (Scorpaeniformes: Liparidae) from the mariana trench. Zootaxa 4358, 161-177. doi: 10.11646/zootaxa.4358.1.7

Gerringer, M. E., Popp, B. N., Linley, T. D., Jamieson, A. J., and Drazen, J. C. (2017b). Comparative feeding ecology of abyssal and hadal fishes through stomach content and amino acid isotope analysis. Deep Sea Res. I 121, 110-120. doi: 10.1016/j.dsr.2017.01.003

Ghanbari, M., Kneifel, W., and Domig, K. J. (2015). A new view of the fish gut microbiome: advances from next-generation sequencing. Aquaculture 448, 464-475. doi: 10.1016/j.aquaculture.2015.06.033

Glass, J. I., Lefkowitz, E. J., Glass, J. S., Heiner, C. R., Chen, E. Y., and Cassell, G. H. (2000). The complete sequence of the mucosal pathogen Ureaplasma urealyticum. Nature 407, 757-762. doi: 10.1038/35037619

Grissa, I., Vergnaud, G., and Pourcel, C. (2007). CRISPRFinder: a web tool to identify clustered regularly interspaced short palindromic repeats. Nucleic Acids Res. 35, 52-57. doi: 10.1093/nar/gkm360

Haas, B. J., Gevers, D., Earl, A. M., Feldgarden, M., Ward, D. V., Giannoukos, G., et al. (2011). Chimeric 16S rRNA sequence formation and detection in Sanger and 454-pyrosequenced PCR amplicons. Genome Res. 21, 494-504. doi: $10.1101 /$ gr.112730.110
Hara, S., Koike, I., Terauchi, K., Kamiya, H., and Tanoue, E. (1996). Abundance of viruses in deep oceanic waters. Mar. Ecol. Prog. Ser. 145, 269-277. doi: 10.3354/meps145269

He, L. S., Zhang, P. W., Huang, J. M., Zhu, F. C., Danchin, A., and Wang, Y. (2017). The enigmatic genome of an obligate ancient Spiroplasma symbiont in a hadal holothurian. Appl. Environ. Microbiol. 84:e1965-17. doi: 10.1128/AEM. 01965-17

Hongoh, Y., Ohkuma, M., and Kudo, T. (2003). Molecular analysis of bacterial microbiota in the gut of the termite Reticulitermes speratus (Isoptera; Rhinotermitidae). FEMS Microbiol. Ecol. 44, 231-242. doi: 10.1016/S01686496(03)00026-6

Indikova, I., Much, P., Stipkovits, L., Siebert-Gulle, K., Szostak, M. P., Rosengarten, R., et al. (2013). Role of the GapA and CrmA cytadhesins of Mycoplasma gallisepticum in promoting virulence and host colonization. Infect. Immun. 81, 1618-1624. doi: 10.1128/iai.00112-13

Jamieson, A. J., Fujii, T., Mayor, D. J., Solan, M., and Priede, I. G. (2010). Hadal trenches: the ecology of the deepest places on Earth. Trends Ecol. Evol. 25, 190-197. doi: 10.1016/j.tree.2009.09.009

Jensen, J. S., Blom, J., and Lind, K. (1994). Intracellular location of Mycoplasma genitalium in cultured Vero cells as demonstrated by electron microscopy. Int. J. Exp. Pathol. 75, 91-98.

Jorgensen, B. B., and Boetius, A. (2007). Feast and famine-microbial life in the deep-sea bed. Nat. Rev. Microbiol. 5, 770-781. doi: 10.1038/nrmicro1745

Kannan, T. R., and Baseman, J. B. (2006). ADP-ribosylating and vacuolating cytotoxin of Mycoplasma pneumoniae represents unique virulence determinant among bacterial pathogens. Proc. Natl Acad. Sci. U.S.A. 103, 6724-6729. doi: 10.1073/pnas.0510644103

Kessel, M. A. V., Dutilh, B. E., Neveling, K., Kwint, M. P., Veltman, J. A., Flik, G., et al. (2011). Pyrosequencing of $16 \mathrm{~S}$ rRNA gene amplicons to study the microbiota in the gastrointestinal tract of carp (Cyprinus carpio L.). AMB Express 1:41. doi: 10.1186/2191-0855-1-41

Kokkayil, P., and Dhawan, B. (2015). Ureaplasma: current perspectives. Indian J. Med. Microbiol. 33, 205-214. doi: 10.4103/0255-0857.154850

Land, M. L., Locascio, P. F., Chen, G. L., Doug, H., Larimer, F. W., and Hauser, L. J. (2010). Prodigal: prokaryotic gene recognition and translation initiation site identification. BMC Bioinformatics 11:119. doi: 10.1186/1471-210511-119

Lara, E., Vaque, D., Sa, E. L., Boras, J. A., Gomes, A., Borrull, E., et al. (2017). Unveiling the role and life strategies of viruses from the surface to the dark ocean. Sci. Adv. 3:e1602565. doi: 10.1126/sciadv.160 2565

Leblanc, J. G., Milani, C., de Giori, G. S., Sesma, F., Van, S. D., and Ventura, M. (2013). Bacteria as vitamin suppliers to their host: a gut microbiota perspective. Curr. Opin. Biotech. 24, 160-168. doi: 10.1016/j.copbio.2012.08.005

Leclercq, S., Dittmer, J., Bouchon, D., and Cordaux, R. (2014). Phylogenomics of "candidatus hepatoplasma crinochetorum," a lineage of mollicutes associated with noninsect arthropods. Genome Biol. Evol. 6, 407-415. doi: 10.1093/gbe/ evu020

Ley, R. E., Lozupone, C. A., Hamady, M., Knight, R., and Gordon, J. I. (2008). Worlds within worlds: evolution of the vertebrate gut microbiota. Nat. Rev. Microbiol. 6, 776-788. doi: 10.1038/nrmicro1978

Li, H., Handsaker, B., Wysoker, A., Fennell, T., Ruan, J., Homer, N., et al. (2009). The sequence alignment/map format and SAMtools. Bioinformatics 25, 2078 2079. doi: 10.1093/bioinformatics/btp352

Linley, T. D., Gerringer, M. E., Yancey, P. H., Drazen, J. C., Weinstock, C. L., and Jamieson, A. J. (2016). Fishes of the hadal zone including new species, in situ observations and depth records of Liparidae. Deep Sea Res. I 114, 99-110. doi: 10.1016/j.dsr.2016.05.003

Lowe, T. M., and Eddy, S. R. (1997). tRNAscan-SE: a program for improved detection of transfer RNA genes in genomic sequence. Nucleic Acids Res. 25, 955-964. doi: 10.1093/nar/25.5.955

Makarova, K. S., Wolf, Y. I., Alkhnbashi, O. S., Costa, F., Shah, S. A., Saunders, S. J., et al. (2015). An updated evolutionary classification of CRISPR-Cas systems. Nat. Rev. Microbiol. 13, 722-736. doi: 10.1038/nrmicro3569

Moran, N. A., Dale, C., Dunbar, H., Smith, W. A., and Ochman, H. (2003). Intracellular symbionts of sharpshooters (Insecta: Hemiptera: Cicadellinae) form a distinct clade with a small genome. Environ. Microbiol. 5, 116-126. doi: 10.1046/j.1462-2920.2003.00391.x 
Nicholson, J. K., Holmes, E., Kinross, J., Burcelin, R., Gibson, G., Jia, W., et al. (2012). Host-gut microbiota metabolic interactions. Science 336, 1262-1267. doi: 10.1126/science. 1223813

Nunoura, T., Takaki, Y., Hirai, M., Shimamura, S., Makabe, A., Koide, O., et al. (2015). Hadal biosphere: insight into the microbial ecosystem in the deepest ocean on Earth. Proc. Natl Acad. Sci. U.S.A. 112, E1230-E1236. doi: 10.1073/ pnas. 1421816112

Ogata, H., Goto, S., Sato, K., Fujibuchi, W., Bono, H., and Kanehisa, M. (2000). KEGG: kyoto encyclopedia of genes and genomes. Nucleic Acids Res. 27, 29-34. doi: 10.1093/nar/28.1.27

Paniagua Voirol, L. R., Frago, E., Kaltenpoth, M., Hilker, M., and Fatouros, N. E. (2018). Bacterial symbionts in Lepidoptera: their diversity, transmission, and impact on the host. Front. Microbiol. 9:556. doi: 10.3389/fmicb.2018.00556

Parks, D. H., Imelfort, M., Skennerton, C. T., Hugenholtz, P., and Tyson, G. W. (2015). CheckM: assessing the quality of microbial genomes recovered from isolates, single cells, and metagenomes. Genome Res. 25, 1043-1055. doi: 10. 1101/gr.186072.114

Patel, R. K., and Jain, M. (2012). NGS QC toolkit: a toolkit for quality control of next generation sequencing data. PLoS One 7:e30619. doi: 10.1371/journal. pone.0030619

Polzin, J., Arevalo, P., Nussbaumer, T., Polz, M. F., and Bright, M. (2019). Polyclonal symbiont populations in hydrothermal vent tubeworms and the environment. Proc. Biol. Sci. 286:20181281. doi: 10.1098/rspb.2018.1281

Pritchard, R. E., Prassinos, A. J., Osborne, J. D., Raviv, Z., and Balish, M. F. (2014). Reduction of hydrogen peroxide accumulation and toxicity by a catalase from Mycoplasma iowae. PLoS One 9:e105188. doi: 10.1371/journal.pone. 0105188

Rawls, J. F., Samuel, B. S., and Gordon, J. I. (2004). Gnotobiotic zebrafish reveal evolutionarily conserved responses to the gut microbiota. Proc. Natl Acad. Sci. U.S.A. 101, 4596-4601. doi: 10.1073/pnas.0400706101

Robillard, G. T., and Broos, J. (1999). Structure/function studies on the bacterial carbohydrate transporters, enzymes II, of the phosphoenolpyruvate-dependent phosphotransferase system. BBA Biomembranes 1422, 73-104. doi: 10.1016/ s0304-4157(99)00002-7

Roeselers, G., Mittge, E. K., Stephens, W. Z., Parichy, D. M., Cavanaugh, C. M., Guillemin, K., et al. (2011). Evidence for a core gut microbiota in the zebrafish. Isme J. 5, 1595-1608. doi: 10.1038/ismej.2011.38

Silvestro, D., and Michalak, I. (2012). raxmlGUI: a graphical front-end for RAxML. Org. Divers. Evol. 12, 335-337. doi: 10.1007/s13127-011-0056-0

Somero, G. N. (1992). Adaptations to high hydrostatic pressure. Annu. Rev. Physiol. 54, 557-577. doi: 10.1146/annurev.ph.54.030192.003013

Stein, D. L. (2005). Descriptions of four new species, redescription of Paraliparis membranaceus, and additional data on species of the fish family Liparidae (Pisces, Scorpaeniformes) from the west coast of South America and the Indian Ocean. Zootaxa 1019, 1-25. doi: 10.11646/zootaxa.1019.1.1

Sverdlov, A. V., Sergei, S., Rao, B. S., Nikolskaya, A. N., Mekhedov, S. L., Raja, M., et al. (2003). The COG database: an updated version includes eukaryotes. BMC Bioinformatics 4:41. doi: 10.1186/1471-2105-4-41
Szczepanek, S. M., Frasca, S. Jr., Schumacher, V. L., Liao, X., Padula, M., Djordjevic, S. P., et al. (2010). Identification of lipoprotein MslA as a neoteric virulence factor of Mycoplasma gallisepticum. Infect. Immun. 78, 3475-3483. doi: 10.1128/ iai.00154- 10

Vander, W. C., Piérard, A., Kley-Raymann, M., and Haas, D. (1984). Pseudomonas aeruginosa mutants affected in anaerobic growth on arginine: evidence for a four-gene cluster encoding the arginine deiminase pathway. J. Bacteriol. 160, 928-934.

Wang, K., Shen, Y., Yang, Y., Gan, X., Liu, G., Hu, K., et al. (2019). Morphology and genome of a snailfish from the Mariana Trench provide insights into deep-sea adaptation. Nat Ecol Evol. 3, 823-833. doi: 10.1038/s41559-019-0864-8

Wang, Y., Huang, J. M., Wang, S. L., Gao, Z. M., Zhang, A. Q., Danchin, A., et al. (2016). Genomic characterization of symbiotic mycoplasmas from the stomach of deep-sea isopod Bathynomus sp. Environ. Microbiol. 18, 2646-2659. doi: 10.1111/1462-2920.13411

Wong, S., Waldrop, T., Summerfelt, S., Davidson, J., Barrows, F., Kenney, P. B., et al. (2013). Aquacultured rainbow trout (Oncorhynchus mykiss) possess a large core intestinal microbiota that is resistant to variation in diet and rearing density. Appl. Environ. Microbiol. 79, 4974-4984. doi: 10.1128/AEM. 00924-13

Wu, M., and Eisen, J. A. (2008). A simple, fast, and accurate method of phylogenomic inference. Genome Biol. 9:R151. doi: 10.1186/gb-2008-9-10r151

Yancey, P. H., Gerringer, M. E., Drazen, J. C., Rowden, A. A., and Jamieson, A. (2014). Marine fish may be biochemically constrained from inhabiting the deepest ocean depths. Proc. Natl Acad. Sci. U.S.A. 111, 4461-4465. doi: 10.1073/ pnas.1322003111

Yoshida, M., Takaki, Y., Eitoku, M., Nunoura, T., and Takai, K. (2013). Metagenomic analysis of viral communities in (hado)pelagic sediments. PLoS One 8:e57271. doi: 10.1371/journal.pone.0057271

Zhang, W., Tian, R. M., and Sun, J. (2018). Genome reduction in Psychromonas species within the gut of an amphipod from the ocean's deepest point. mSystems 3:e00009-18. doi: 10.1128/mSystems.00009-18

Zhang, Y., Heidrich, N., Ampattu, B. J., Gunderson, C. W., Seifert, H. S., Schoen, C., et al. (2013). Processing-independent CRISPR RNAs limit natural transformation in Neisseria meningitidis. Mol. Cell. 50, 488-503. doi: 10.1016/j. molcel.2013.05.001

Conflict of Interest: The authors declare that the research was conducted in the absence of any commercial or financial relationships that could be construed as a potential conflict of interest.

Copyright (c) 2020 Lian, Yan, Huang, Danchin, Wang and He. This is an open-access article distributed under the terms of the Creative Commons Attribution License (CC BY). The use, distribution or reproduction in other forums is permitted, provided the original author(s) and the copyright owner(s) are credited and that the original publication in this journal is cited, in accordance with accepted academic practice. No use, distribution or reproduction is permitted which does not comply with these terms. 